\title{
Repercusiones del tratamiento psicoterapéutico sobre indicadores psico- somáticos en el paciente renal en hemodiálisis
}

\author{
Rosa María Salas Muñoz, Antonio José Fernández Jiménez \\ Centro de Diálisis Sierra Este. Sevilla. España
}

\section{Resumen}

Introducción: Aunque las relaciones psico-somáticas y los efectos del tratamiento psicológico en el desarrollo de enfermedades ha sido un tema de interés en las últimas décadas, no existen estudios con pacientes renales en hemodiálisis.

Objetivo: Evaluar las repercusiones psicológicas y físicas de la aplicación de psicoterapia en el paciente renal en hemodiálisis.

Material y Método: Estudio cuasi experimental. 39 personas recibieron atención psicológica entre 3 y 18 meses. Se les aplicaron los cuestionarios de depresión de Beck y Ansiedad-Rasgo antes y después del tratamiento con psicoterapia, y mientras duró dicho tratamiento también se registraron los valores de frecuencia cardiaca, tensión arterial, peso seco y ganancia hídrica inter-sesiones.

Resultados: Tras el tratamiento psicológico los niveles de depresión descendieron en todos los pacientes excepto en aquellos con depresión leve, y en el caso de la ansiedad, tan sólo descendió en los pacientes con ansiedad elevada. La única variable fisiológica que mostró variaciones durante el tratamiento psicológico fue la ganancia hídrica inter-diálisis, que describió una tendencia lineal descendiente en 24 casos.

Conclusiones: Las repercusiones del tratamiento psicológico pueden verse reflejadas en una variación de los

\section{Correspondencia:}

Rosa María Salas Muñoz

Centro de Diálisis Sierra Este

Avenida de la Innovación, 2.41020 Sevilla

E-mail: psicologia@dialisisandaluza.es niveles de ansiedad y depresión, así como en una disminución de las ganancias de líquido interdiálisis.

PALABRAS CLAVE: hemodiálisis; ansiedad; depresión; balance hidroelectrolítico; psicoterapia.

\section{Impact of psychotherapeutic treatment of psychosomatic indicators in hemodialysis patients}

Introduction: Although psychosomatic relationships and the effects of psychological treatment in the development of diseases has been a topic of interest in recent decades, there are no studies with renal patients on hemodialysis.

Objective: To evaluate the psychological and physical repercussions of the application of psychotherapy in renal patients on hemodialysis.

Material and Method: Quasi-experimental study. 39 people received psychological attention between 3 and 18 months. Beck depression and anxiety-trait questionnaires were applied before and after the treatment with psychotherapy, and during treatment the values of heart rate, blood pressure, dry weight and inter-session water gain were also recorded.

Results: After psychological treatment depression levels decreased in all patients except those with mild depression, and in the case of anxiety, decreased only in patients with high anxiety. The only physiological variable that showed variations during the psychological treatment was the inter-dialysis water gain, which described a descending linear trend in 24 cases. 
Conclusions: The impact of psychological treatment can be reflected in a change in levels of anxiety and depression as well as a decrease in inter-dialytic water gain.

KEYWORDS: hemodialysis; anxiety; depression, water-electrolyte balance; psychotherapy.

\section{Introducción}

Las estrechas inter-relaciones que existen entre aspectos psicológicos y físicos en el ser humano han sido objeto de estudio desde hace décadas no sólo para psicólogos y psiquiatras, sino que también han despertado el interés de profesionales de otras especialidades médicas ${ }^{1-3}$. A día de hoy, la hipótesis que cobra más fuerza es que, más que una relación "causa-efecto" entre alteraciones psicológicas y físicas, lo que tiene lugar es una ruptura en el equilibrio u homeostasis necesario para un funcionamiento saludable de la persona, que puede producirse bien por agentes externos o internos a la misma, y cuyas consecuencias aparecen en forma de alteraciones emocionales, mentales y/o físicas que coexisten y que, desde el punto de vista sanitario, a efectos prácticos, son clasificadas bajo diferentes categorías y diagnósticos que llamamos enfermedades.

Actualmente, no conocemos estudios dedicados a indagar sobre estas relaciones psico-somáticas en personas que padecen insuficiencia renal crónica, a pesar de ser una enfermedad que acapara un alto porcentaje del gasto sanitario de nuestro país ${ }^{4}$, cuyo padecimiento tiene repercusiones importantes en la calidad de vida percibida por los propios pacientes, y que afecta también negativamente al ámbito familiar de los mis$\operatorname{mos}^{5,6}$. Como punto de partida, contamos con literatura que demuestra que su presencia se relaciona, en un alto porcentaje de casos, con trastornos psicológicos, especialmente ansiedad y depresión ${ }^{7-9}$. Además, existe literatura que nos muestra las estrechas relaciones existentes entre la insuficiencia renal y el funcionamiento cardiovascular, resultando la frecuencia cardíaca y la tensión arterial medidas sensibles ante este hecho $0^{10,11}$.

Otros indicadores somáticos estrechamente unidos a la insuficiencia renal son el peso del paciente y la ganancia de líquido interdiálisis, que en la práctica clínica diaria presentan una mayor dificultad para su control ${ }^{12}$. Esto se debe a que, a pesar de que se presupone la capacidad del paciente para influir a voluntad en su control, a través de la ingesta de alimentos y líquidos, lo cierto es que ésta no es tarea fácil para ellos y encuentran grandes dificultades para conseguirlo, llegando a ser la ganancia hídrica inter-sesiones uno de los indicadores más fiables de adherencia al tratamiento de las personas en hemodiálisis ${ }^{13,14}$.

A la luz de estas relaciones psico-somáticas, consideramos que la terapia psicológica podría convertirse en un complemento de gran valor para el tratamiento médico/farmacológico del paciente renal, y que podría tener repercusiones no sólo sobre parámetros cognitivos y emocionales, sino también fisiológicos, mejorando su estado general de salud. Sin embargo, si bien existen múltiples estudios que aportan información empírica sobre el estado físico y psicológico de personas con insuficiencia renal en hemodiálisis, como los mencionados anteriormente, actualmente no contamos con datos acerca de la influencia del tratamiento psicológico en este colectivo.

Por tanto, el objetivo del estudio consistió en evaluar las repercusiones psicológicas y físicas de la aplicación de psicoterapia en el paciente renal en hemodiálisis.

\section{Material y Método}

Se realizó estudio cuasi-experimental de 18 meses de duración (octubre de 2016 a marzo de 2018).

La obtención de la muestra se realizó en dos fases. En un primer lugar se incluyeron a todos los pacientes en hemodiálisis del centro Diálisis Andaluza (Sevilla), mayores de edad, que dieron su consentimiento informado para participar en el estudio. A estos participantes se les realizó una entrevista para recoger información sobre: 1) datos biográficos, 2) situación actual bio-psico-social, 3) rasgos destacados psicológicos y comportamentales y 4) principales estrategias de afrontamiento y auto-apoyo. Además, se les administraron el cuestionario de Depresión de Beck para pacientes médicos $\left(\right.$ BDI-FS) ${ }^{15}$ y el cuestionario de Ansiedad Estado-Rasgo $(\mathrm{STAI})^{16}$.

En una segunda fase, se llevó a cabo la selección de aquellos pacientes que cumplieran al menos con uno de los siguientes criterios:

- Que existiera la demanda de atención psicológica por parte del propio paciente. 
- Que la puntuación obtenida en la escala estado-rasgo del STAI fuera igual o superior a 7.

- Que la puntuación obtenida en inventario de depresión BDI-FS fuera igual o superior a 9.

- Que por parte del área de enfermería nefrológica y/o nutrición del centro de diálisis, se reportara que la persona refiere dificultades para controlar la ingesta de líquidos.

- Que los indicadores de ganancia hídrica inter-sesiones fueran iguales o superiores a $3,5 \mathrm{~kg}$ en más de 3 ocasiones en el último mes.

- Que en la entrevista realizada hubieran referido la presencia de algún evento estresante en sus vidas que estuviera interfiriendo significativamente en su funcionamiento cotidiano.

\section{Intervención con psicoterapia:}

Las personas seleccionadas recibieron atención psicológica, por parte de la psicóloga del centro de diálisis, en forma de sesiones de psicoterapia de orientación humanista (terapia Gestalt), siendo esta una corriente de la psicología humanista cuyo creador, Fritz Perls, desarrolló en la década de los 40 y que pone el énfasis en el desarrollo de la persona a través del vínculo entre terapeuta y paciente, basándose en tres pilares fundamentales: 1) el "darse cuenta" o toma de conciencia, 2) vivir el presente "aquí y ahora" y 3) fomentar la responsabilidad sobre el propio proceso de crecimiento y sobre los acontecimientos que se experimentan a lo largo del ciclo vital17. La terapia se llevaba a cabo con una frecuencia de al menos una vez por semana. Las sesiones tuvieron una duración variable, que fluctuó entre 30 y 50 minutos, y tenían lugar bien antes de que la persona entrara en la sala para recibir el tratamiento de hemodiálisis, o bien en la propia sala de diálisis mientras estaban dializándose. El número de sesiones de psicoterapia varió en cada caso, según las necesidades de cada persona.

Como criterio de retirada, no se estudiaron aquellos participantes que hubieran recibido menos de 6 sesiones de psicoterapia.

\section{Variables estudiadas:}

Se recogieron las variables correspondientes a los cuestionarios de ansiedad (STAI) y depresión (BDI-FS) antes y tras el periodo de sesiones con psicoterapia. Así mismo, antes de cada sesión de diálisis y durante el tiempo que duró el tratamiento psicológico, se recogieron los siguientes parámetros fisiológicos: peso seco, ganancia de peso interdiálisis, presión arterial y frecuencia cardiaca.

\section{Instrumentos de medida:}

- Inventario de Depresión de Beck para pacientes médicos $(B D I-F S)^{15}$. Consta de 7 ítems, extraídos del BDI-II, para evaluar depresión en adolescentes y adultos con un diagnóstico médico. Permite conocer los síntomas cognitivos y afectivos vinculados a la depresión, excluyendo aquellos que podrían deberse a trastornos o enfermedades médicas evitando así falsos positivos. Las puntuaciones arrojadas por este inventario se dividieron en cuatro tramos de depresión establecidos por los propios autores del mismo: 1) 0-3, mínima. 2) 4-8, leve. 3) 9-12, moderada. 4) 13-21, grave.

- Cuestionario de Ansiedad Estado-Rasgo (STAI) ${ }^{16}$. Se tuvo en cuenta la puntuación de la escala Ansiedad-Rasgo, ya que señala una propensión ansiosa más estable que la escala Ansiedad-Estado, que refleja un estado emocional circunscrito al momento de la evaluación. Los puntajes de este cuestionario para cada sujeto quedan transformados en un único valor decatípico que va de 1 a 10. Para mayor facilidad interpretativa, hemos considerado la agrupación de las puntuaciones de la muestra atendiendo a tres tramos de ansiedad: 1) 1-4, baja. 2) 5-7 moderada. 3) 8-10 elevada.

\section{Aspectos éticos}

La información recogida ha sido tratada con arreglo a la actual Ley Orgánica 15/1999 de 13 diciembre, sobre "Protección de datos de carácter personal", y con el único fin del desarrollo de esta investigación. El estudio fue aprobado por el centro Diálisis Andaluza, de Sevilla. Se solicitó el consentimiento informado a todos los participantes en el estudio.

\section{Análisis estadístico}

Se realizó un análisis descriptivo de frecuencias para los datos de depresión y ansiedad pre- y post-tratamiento psicológico, atendiendo a los diferentes intervalos de puntuación establecidos. Las medias obtenidas con los datos pre- y post-tratamiento para depresión y ansiedad, se compararon mediante el estadístico T de Student para muestras relacionadas.

Los datos de cada sujeto para cada una de las variables fisiológicas objeto de estudio, por separado, fueron so- 
metidos a un análisis de tendencias mediante regresión lineal simple.

En todos los casos, se consideró una $p$ con valor $<0,05$ como estadísticamente significativa.

\section{Resultados}

\section{Datos demográficos}

De los 103 pacientes iniciales se obtuvo una muestra final de 39 sujetos que cumplieron los criterios de inclusión para este estudio ( 21 mujeres y 18 hombres), con edades comprendidas entre los 26 y los 80 años.

\section{Resultados BDI-FS}

Los porcentajes de las puntuaciones obtenidas pre y post-tratamiento, agrupadas por intervalos, pueden observarse en la Figura 1. En la Tabla 1 aparecen las puntuaciones individuales para cada uno de los sujetos que recibieron tratamiento psicológico, antes y después del mismo.

El estadístico T de Student para muestras relacionadas no arrojó diferencia estadísticamente significativa tras la aplicación de este cuestionario pre- (media 5,82) y post- tratamiento (media 5,46) para un intervalo de confianza del $95 \%(-0,69 ; 1,41)$, obteniéndose una $p=0,493$.

\section{Resultados STAI}

En la Figura 2, pueden observarse los porcentajes para cada tramo de puntuaciones de ansiedad pre y post-tratamiento. En la Tabla 1, pueden apreciarse los decatipos arrojados por este cuestionario, para cada sujeto, antes y después del tratamiento psicológico.

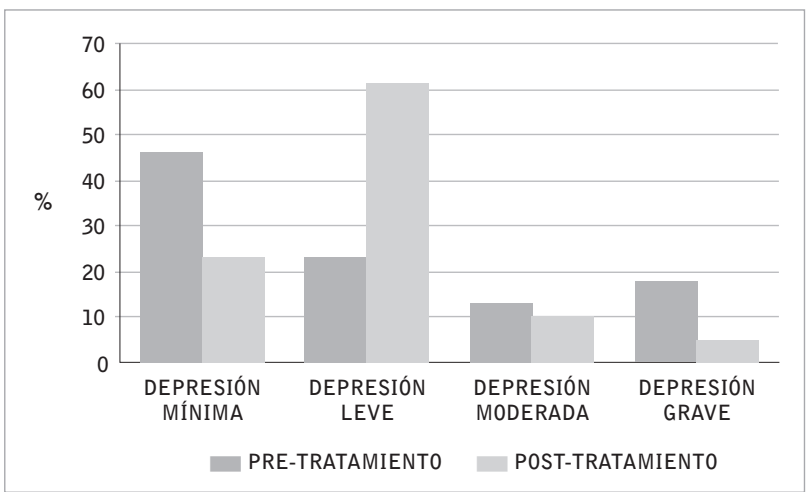

Figura 1. Prevalencia de grado de depresión antes y después de la intervención según el cuestionario BDI-FS.

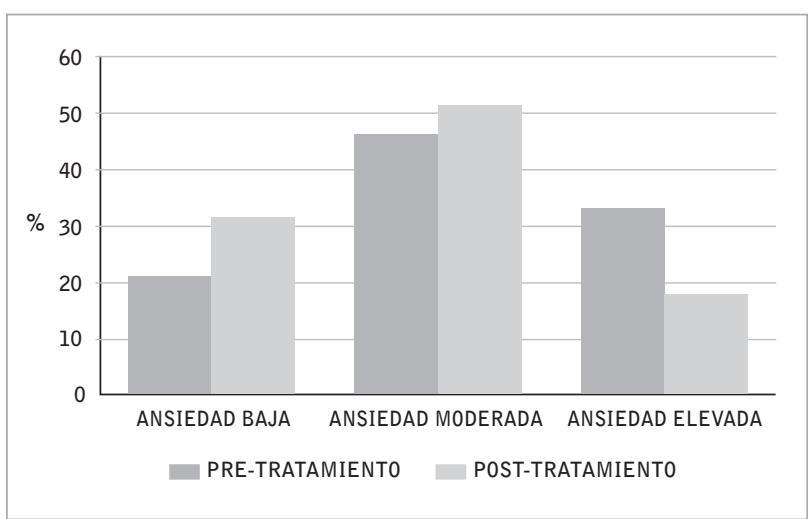

Figura 2. Prevalencia de grado de ansiedad antes y después de la intervención según el cuestionario STAI.

La comparación de los datos de este cuestionario, obtenidos antes (media 6,64) y después (media 5,72) del tratamiento psicológico, para el grupo de pacientes seleccionados, no resultó estadísticamente significativa (IC95\% -0,07-1,92; $p=0,07$ ).

\section{Parámetros fisiológicos}

El tiempo que cada paciente estuvo en tratamiento psicológico (a razón de una sesión semanal) fue variable, oscilando entre 3 meses en el caso de menor duración, y 18 meses en el caso donde la psicoterapia se prolongó por más tiempo (duración total de este estudio), por lo que el número de registros obtenido de los parámetros fisiológicos estudiados osciló entre 36 registros en el caso de menor duración y de 239 registros en el caso más prolongado, coincidiendo con el número de sesiones de hemodiálisis recibidas durante dicho período. EI análisis de tendencia para las variables de peso seco, tensión arterial y frecuencia cardiaca pre-diálisis no mostró un patrón predominante en ningún caso.

En lo que respecta a la ganancia hídrica inter-sesiones, pudo observarse un comportamiento lineal descendiente en un $71,79 \%$ de los casos estudiados (28 pacientes). Dicha tendencia resultó estadísticamente significativa en el $75 \%$ de estos casos (21 pacientes). En la Tabla 2, puede observarse un resumen de estos resultados para cada sujeto. Por el contrario, un $17,94 \%$ de los pacientes que recibieron tratamiento psicológico ( $7 \mathrm{su}$ jetos), mostraron ganancias hídricas inter-sesiones con un comportamiento ascendente, que resultó significativo en 3 de estos casos. Por último, en el 10,25\% de los pacientes (4 sujetos), los valores de ganancias hídricas se mantuvieron estables durante el tiempo que duró el tratamiento psicológico. 
Tabla 1. Resultados depresión y ansiedad pre y post tratamiento psicológico.

\begin{tabular}{|c|c|c|c|c|c|c|}
\hline \multirow{2}{*}{$\begin{array}{l}\text { Sujeto } \\
1\end{array}$} & \multirow{2}{*}{$\begin{array}{c}\text { Puntuación } \\
\text { BDI-FS pre } \\
13\end{array}$} & \multicolumn{2}{|c|}{$\begin{array}{l}\text { Puntuación } \\
\text { BDI-FS post }\end{array}$} & \multirow{2}{*}{$\begin{array}{c}\text { Puntuación } \\
\text { STAI pre } \\
9\end{array}$} & \multicolumn{2}{|c|}{$\begin{array}{l}\text { Puntuaciór } \\
\text { STAI post }\end{array}$} \\
\hline & & $\downarrow$ & 8 & & $\downarrow$ & 4 \\
\hline 2 & & $\downarrow$ & & & & \\
\hline & 13 & 10 & & 9 & $\downarrow$ & 4 \\
\hline 3 & & $\downarrow$ & & & & \\
\hline & 12 & 10 & & 9 & $\downarrow$ & 4 \\
\hline 4 & 11 & $\downarrow$ & 8 & 9 & $\downarrow$ & 5 \\
\hline 5 & 3 & $\downarrow$ & 2 & 10 & $\downarrow$ & 6 \\
\hline 6 & 10 & $\downarrow$ & 5 & 9 & $\downarrow$ & 5 \\
\hline 7 & & $\downarrow$ & & & & \\
\hline & 17 & 14 & & 9 & $\downarrow$ & 2 \\
\hline 8 & 16 & $\begin{array}{c}\downarrow \\
13\end{array}$ & & 10 & $\downarrow$ & 3 \\
\hline 9 & 13 & $\downarrow$ & 8 & 10 & $\downarrow$ & 6 \\
\hline 10 & 0 & $\uparrow$ & 2 & 2 & $\uparrow$ & 6 \\
\hline 11 & 4 & $\uparrow$ & 5 & 6 & $\uparrow$ & 8 \\
\hline 12 & 0 & $\uparrow$ & 2 & 4 & $\uparrow$ & 7 \\
\hline 13 & 0 & $\uparrow$ & 3 & 4 & $\uparrow$ & 6 \\
\hline 14 & 0 & $\uparrow$ & 4 & 2 & $\uparrow$ & 5 \\
\hline 15 & 1 & $\uparrow$ & 4 & 4 & $\uparrow$ & 9 \\
\hline 16 & 1 & $\uparrow$ & 5 & 4 & $\uparrow$ & 5 \\
\hline 17 & 3 & $\downarrow$ & 1 & 6 & $\downarrow$ & 8 \\
\hline 18 & 6 & $\downarrow$ & 4 & 6 & $\downarrow$ & 7 \\
\hline 19 & 13 & $\downarrow$ & 8 & 9 & $\downarrow$ & 10 \\
\hline 20 & 3 & $\downarrow$ & 3 & 8 & $\leftrightarrow$ & 8 \\
\hline 21 & 3 & $\downarrow$ & 2 & 6 & $\leftrightarrow$ & 6 \\
\hline 22 & 15 & $\downarrow$ & 6 & 8 & $\leftrightarrow$ & 8 \\
\hline 23 & 6 & $\downarrow$ & 4 & 7 & $\leftrightarrow$ & 7 \\
\hline 24 & 9 & $\downarrow$ & 5 & 7 & $\leftrightarrow$ & 7 \\
\hline 25 & 10 & $\downarrow$ & 6 & 7 & $\leftrightarrow$ & 7 \\
\hline 26 & 0 & $\uparrow$ & 4 & 7 & $\downarrow$ & 2 \\
\hline 27 & 0 & $\uparrow$ & 4 & 7 & $\downarrow$ & 4 \\
\hline 28 & 2 & $\uparrow$ & 4 & 5 & $\downarrow$ & 3 \\
\hline 29 & 4 & $\uparrow$ & 5 & 3 & $\downarrow$ & 4 \\
\hline 30 & 2 & $\uparrow$ & 6 & 7 & $\downarrow$ & 4 \\
\hline 31 & 5 & $\uparrow$ & 6 & 5 & $\downarrow$ & 3 \\
\hline 32 & 2 & $\uparrow$ & 4 & 5 & $\downarrow$ & 4 \\
\hline 33 & 8 & $\uparrow$ & 9 & 10 & $\downarrow$ & 6 \\
\hline 34 & 0 & $\uparrow$ & 4 & 5 & $\leftrightarrow$ & 5 \\
\hline 35 & 2 & $\uparrow$ & 3 & 6 & $\leftrightarrow$ & 6 \\
\hline 36 & 8 & $\uparrow$ & 9 & 7 & $\leftrightarrow$ & 7 \\
\hline 37 & 5 & $\uparrow$ & 6 & 7 & $\leftrightarrow$ & 7 \\
\hline 38 & 2 & $\leftrightarrow$ & 2 & 4 & $\uparrow$ & 8 \\
\hline 39 & 5 & $\leftrightarrow$ & 5 & 7 & $\leftrightarrow$ & 7 \\
\hline
\end{tabular}

$\uparrow$ La puntuación asciende tras el tratamiento psicológico.

$\downarrow$ La puntuación desciende tras el tratamiento psicológico.

$\leftrightarrow$ La puntuación se mantiene tras el tratamiento psicológico.
Tabla 2. Resultados regresión lineal con tendencia descendiente para datos de ganancia hídrica interdiálisis.

\begin{tabular}{|c|c|c|c|}
\hline Sujeto & $\begin{array}{l}\text { Sesiones } \\
\text { diálisis }\end{array}$ & $\begin{array}{l}\text { Coeficiente } \\
\text { Regresión }\end{array}$ & $\begin{array}{l}\text { Significación } \\
\text { Estadística }\end{array}$ \\
\hline 1 & 36 & $b l=-0,26$ & $p=0,039$ * \\
\hline 2 & 63 & $b l=-0,16$ & $p=0,004^{*}$ \\
\hline 3 & 57 & $b l=-0,11$ & $p=0,007^{*}$ \\
\hline 4 & 67 & $b l=-0,009$ & $p=0,048^{*}$ \\
\hline 5 & 44 & $b l=-0,048$ & $p=0,000$ * \\
\hline 6 & 92 & $b l=-0,008$ & $p=0,021^{*}$ \\
\hline 7 & 231 & $b l=-0,002$ & $p=0,001^{*}$ \\
\hline 8 & 91 & $b l=-0,012$ & $p=0,000^{*}$ \\
\hline 9 & 74 & $b l=-0,014$ & $p=0,003$ * \\
\hline 10 & 71 & $b l=-0,04$ & $p=0,000^{*}$ \\
\hline 11 & 75 & $b l=-0,17$ & $p=0,000^{*}$ \\
\hline 12 & 93 & $b l=-0,014$ & $p=0,000^{*}$ \\
\hline 13 & 89 & $b l=-0,009$ & $p=0,021^{*}$ \\
\hline 14 & 75 & $b l=-0,011$ & $p=0,006^{*}$ \\
\hline 15 & 91 & $b l=-0,01$ & $p=0,000^{*}$ \\
\hline 16 & 89 & $b l=-0,009$ & $p=0,000^{*}$ \\
\hline 17 & 101 & $b l=-0,007$ & $\mathrm{p}=0,001^{*}$ \\
\hline 18 & 65 & $b l=-0,014$ & $p=0,017^{*}$ \\
\hline 19 & 57 & $b l=-0,016$ & $p=0,034^{*}$ \\
\hline 20 & 115 & $b l=-0,006$ & $p=0,03 *$ \\
\hline 21 & 86 & $b l=-0,009$ & $p=0,038^{*}$ \\
\hline 22 & 78 & $b l=-0,024$ & $p=0,292$ \\
\hline 23 & 96 & $b l=-0,003$ & $p=0,226$ \\
\hline 24 & 117 & $b l=-0,002$ & $p=0,682$ \\
\hline 25 & 189 & $b l=-0,001$ & $p=0,226$ \\
\hline 26 & 127 & $b l=-0,002$ & $p=0,525$ \\
\hline 27 & 39 & $b l=-0,009$ & $p=0,454$ \\
\hline 28 & 63 & $b l=-0,01$ & $p=0,079$ \\
\hline
\end{tabular}

*p estadísticamente significativa bajo intervalo de confianza del 95\%.

\section{Discusión}

Los resultados obtenidos muestran que la intervención psicoterapéutica podría tener relación con cambios en los niveles de ansiedad y depresión de los pacientes en hemodiálisis. Como cabría esperar, observamos un descenso en uno o en ambos valores tras el tratamiento psicológico en la mayoría de los casos. Sin embargo, también hemos podido registrar cómo estos indicadores se mantienen o incluso aumentan sensiblemente después de la intervención psicológica en muchos sujetos. Estos resultados son fruto de un tratamiento 
psicoterapéutico de corte humanista basado fundamentalmente en la Terapia Gestalt ${ }^{17}$. La elección del enfoque terapéutico tuvo lugar considerando no introducir, como parte del tratamiento, tareas o ejercicios de corte cognitivo-conductual, que el paciente pudiera percibir como un aumento en la cantidad de requerimientos y exigencias que se le demandan, y que se sumarían a las que ya su condición de enfermo renal le supone ${ }^{18}$. Además, la Terapia Gestalt tiene como precepto contribuir a la toma de conciencia de la persona de su situación presente, y al contacto con sus propias necesidades, a veces no atendidas por diferentes moti$\operatorname{vos}^{19}$. Este último aspecto, podría ser responsable del sensible aumento en algunos pacientes de los niveles de ansiedad y depresión obtenidos tras la psicoterapia. Pensamos que, más que por un empeoramiento de su estado anímico, esto podría deberse a que la intervención psicológica les ha ayudado a sensibilizarse con su situación y estar más en contacto con los propios sentimientos, que se hacen más presentes y conscientes. Este aspecto es importante para que la persona pueda orientar de forma más saludable sus respuestas ante las demandas internas y externas a las que se ve expuesto, que incluyen las que su condición de paciente renal conlleva, como quedaría reflejado en muchos de estos casos por el descenso paralelo en la ingesta de líquidos entre las sesiones de diálisis.

En este sentido, aunque no se han observado cambios a nivel fisiológico en frecuencias cardíacas, tensiones arteriales y valores de peso seco, sí hemos podido identificar un patrón descendiente en los valores de ganancia hídrica inter-diálisis, de forma que la cantidad de líquido ingerida por muchos de los pacientes, entre una sesión de diálisis y la siguiente, podría ir descendiendo a medida que mejoraban psicológicamente. Esto nos lleva a especular sobre un posible vínculo entre los resultados obtenidos y el desarrollo en estos sujetos, gracias a la psicoterapia, de mejores herramientas de gestión emocional y control de impulsos, incluyendo el relacionado con la ingesta de líquidos, que es una de las restricciones más difíciles de llevar a cabo por las personas en hemodiálisis ${ }^{12}$.

Nuestros resultados se ven avalados por otros estudios que arrojan disminuciones en los índices de depresión de los pacientes en hemodiálisis tras intervenciones psicológicas basadas en otros enfoques terapéuti$\cos ^{20,21}$. Además, se ha descrito la relación entre un aumento en la adherencia al tratamiento de estos pacientes y la intervención psicológica, reflejada en algunos estudios en la mejor gestión de la ingesta de líquidos ${ }^{22-29}$. No obstante, queremos resaltar como diferencia con respecto a nuestro trabajo, que dichas intervenciones estuvieron enfocadas precisamente a introducir un programa de entrenamiento dirigido a que el paciente redujera la ingesta hídrica. El hecho de que no haya sido este el objetivo de la intervención psicológica en nuestro estudio, estando ésta enfocada a trabajar aspectos de índole exclusivamente emocional, deja ver la importancia de la interrelación existente entre indicadores psicológicos y fisiológicos en el paciente renal.

La utilidad de estos resultados para el personal de enfermería nefrológica, podría residir en el hecho de que la observación de ganancias hídricas elevadas inter-diálisis, podría suponer no sólo una llamada de atención sobre el control de la ingesta de líquidos, sino también sobre la necesidad del paciente de atención psicológica. De ser así, este indicador podría convertirse en un referente somático relacionado con el bienestar emocional de la persona en hemodiálisis, hipótesis que requiere de investigaciones más exhaustivas para poder ser confirmada.

A partir de los resultados observados, podríamos concluir que el tratamiento psicológico tiene repercusiones para los pacientes con insuficiencia renal en hemodiálisis, no sólo a nivel psicológico, mejorando en muchos casos los niveles de ansiedad y depresión que estos presentan, sino también a nivel somático, lo cual queda reflejado en el descenso progresivo de los valores de ganancia hídrica inter-diálisis obtenidos a lo largo del tratamiento

Recibido: 28 noviembre 2018

Revisado: 30 enero 2019

Modificado: 18 febrero 2019

Aceptado: 25 febrero 2019 


\section{Bibliografía}

1. Chiozza L. ¿Por qué enfermamos?. La historia que se oculta en el cuerpo. Buenos Aires: El zorzal; 2007.

2. Rof Carballo J. Teoría y Práctica Psicosomática. España: Descleé de Brower; 1984.

3. Rivera L. Medicina Psicosomática. EEUU: Createspace Independent Publishing Platform; 2015.

4. Vargas Marcos F. Documento Marco sobre enfermedad renal crónica (ERC) dentro de la estrategia de abordaje a la cronicidad del sistema nacional de salud. [internet]. España: Ministerio de Sanidad, Servicios Sociales e Igualdad; 2015 [Consultado 10 Jul 2018]. Disponible en: http://www.msssi.gob.es/ organizacion/sns/planCalidadSNS/pdf/Enfermedad Renal_Cronica_2015.pdf

5. Romero Massa E, Rodríguez Castilla J, Pereira Díaz B. El cuidador familiar del paciente renal y su calidad de vida. Sobrecarga y calidad de vida percibida en cuidadores familiares de pacientes renales. [Internet]. Rev Cuba Enferm 2015 [Consultado 10 Jul 2018]; 31(4). Disponible en: www.revenfermeria. sld.cu/index.php/enf/article/view/299/134

6. Martínez L, Valdés YG, Rodríguez R. Estrategias de afrontamiento al estrés en cuidadores de pacientes en hemodiálisis. Enferm Nefrol. 2017;20(2):139-48.

7. García-Llana H, Remor E, Del Peso G, Selgas R. El papel de la depresión, la ansiedad, el estrés y la adhesión al tratamiento en la calidad de vida relacionada con la salud en pacientes en diálisis: revisión sistemática de la literatura. Nefrologia 2014;34(5):637-57.

8. Gómez L, Pac N, Manresa M, Lozano S, Chevarria JL. Prevalencia de ansiedad y depresión en pacientes de hemodiálisis. Enferm Nefrol. 2015;18(2):112-7.

9. Moya MA. Estudio del estado emocional de los pacientes en hemodiálisis. Enferm Nefrol. 2017;20(1):48-56.

10. Quijada MT, Gómez R. Prevalencia y conocimiento de los factores de riesgo cardiovascular en pacientes en tratamiento de diálisis. Enferm Nefrol. 2018;21(2):130-6.
11. González I, Casanova C, Escobar C, Garcia A, Peraira JR, Prieto $E$, et al. Enfermedad cardiovascular y función renal. Mecanismos patogénicos. Rev Esp Cardiol Supl. 2008;8(E):S10-21.

12. Iborra C. Adhesión a la restricción de líquidos en pacientes renales tratados con hemodiálisis. Tesis Doctoral. Alicante: Universidad Miguel Hernández; 2013.

13. Brady B, Tucker C, Alfino P, Tarrant D, Finlayson $G$. An investigation of factors associated with fluid adherence among hemodialysis patients: a self-efficacy theory based approach. Ann Behav Med.1997;19(4):339-43.

14. Fisher L. Psychological factors influencing thirst and drinking in hemodialysis patients on a fluid restriction. Behav Cognit Psychother. 2004;32(3):347-52.

15. Beck AT, Steer RA, Brown GK. Inventario de depresión de Beck Fast-Screen para pacientes médicos. Adaptación española por Sanz J, Izquierdo A y García-Vera MP. España: Pearson; 2011.

16. Spielberg RD, Gorsuch RL, Lushene RE. Cuestionario de Ansiedad Estado-Rasgo STAI. Adaptación española por Buela-Casal G, Guillén-Riquelme A y Seisdedos N. España:TEA Ediciones; 2011.

17. Peñarrubia F. Terapia Gestalt, la vía del vacío fértil. Madrid: Alianza Editorial; 1998.

18. Hernández L. El sufrimiento en el paciente insuficiente renal. Mañongo 2007;15(29):399-414.

19. Ginger $S$ y Ginger A. La Gestalt, una terapia de contacto. México: Manual Moderno; 1993.

20. Cukor D, Ver Halen N, Asher DR, Coplan JD, Weedon J, Wyka KE et al. Psychosocial intervention improves depression, quality of life, and fluid adherence in hemodialysis. J Am Soc Nephrol. 2014;25(1):196-206.

21. Duarte PS, Miyazaki MC, Blay SL, Sesso R. Cognitive-behavioral group therapy is an effective treatment for mayor depression in hemodialysis patients. Kidney Int. 2009;76(4):414-21.

22. Christensen AJ, Moran PJ, Wiebe JS, Ehlers SL, Lawton WJ. Effect of a behavioral self-regulation intervention on patient adherence in hemodialysis. Health Psychol. 2002;21(4):393-7. 
23. Hegel MT, Ayllon T, Theil G, Oulton B. Improving adherence to fluid restrictions in male haemodialysis patients: a comparison of cognitive and behavioural approaches. Health Psychol. 1992;11(5):324-30.

24. Lindberg M, Wikstrom B, Lindberg P. A behavioural nursing intervention for reduced fluid overload in haemodialysis patients. Initial results of acceptability, feasibility and efficacy. J Nurs Healthc Chronic Illn. 2011;3(2):87-98.

25. Mosley TH, Eisen AR, Bruce BK, Brantley PJ, Cocke TB. Contingent social reinforcement for fluid compliance in a hemodialysis patient. J Beh Ther Exp Psychiatry. 1993;24(1):77-81.

26. Casey J, Johnson V, McClelland P. Impact of stepped verbal and written reinforcement of fluid balance advice within an outpatient haemodialysis unit: a pilot study. J Hum Nutr Diet. 2002;15(1):43-7.
27. Nozaki C, Oka M, Chaboyer W. The effects of a cognitive behavioural therapy programme for self-care on haemodialysis patients. Int $\mathrm{J}$ Nurs Pract. 2005;11(5):228-36.

28. Sagawa M, Oka M, Chaboyer W, Satoh W, Yamaguchi M. Cognitive behavioural therapy for fluid control in haemodialysis patients. Nephrol Nurs J. 2001;28:37-9.

29. Sharp J, Wild MR, Gumley AI, Deighan CJ.A cognitive behavioral group approach to enhance adherence to hemodialysis fluid restrictions: a randomized controlled trial. Am J Kidney Dis. 2005;45(6):104657.

Este artículo se distribuye bajo una Licencia Creative Commons Atribución-NoComercial 4.0 Internacional. https://creativecommons.org/licenses/by-nc/4.0/

Open Access (c) (7) \& 